\title{
Adrenal Cortex Ultrastructural Alterations Caused by Zootoxins
}

\author{
M. Pulido-Méndez ${ }^{*}$, A. Rodríguez-Acosta ${ }^{* *}$, and H.J. Finol ${ }^{* * *}$.
}

*Instituto de Medicina Experimental, Facultad de Medicina, Universidad Central de Venezuela, Apartado 47114, Caracas 1041A, Venezuela.

** Instituto de Medicina Tropical, Facultad de Medicina, Universidad Central de Venezuela, Apartado 47423, Caracas 1041 A, Venezuela.

${ }^{* * *}$ Centro de Microscopía Electrónica, Facultad de Ciencias, Universidad Central de Venezuela, Apartado 47114, Caracas 1041A, Venezuela.

The organ ultrastructural pathology and its correlation with clinical manifestations in zootoxins envenoming have not been studied enough. Following scorpion or bee stings, some patients develop clinical symptoms as hypotension, sweating, coldness, tachycardia and shock that additionally to alterations in other systems, could be explained by suprarenal gland dysfunction [1,2]. As far as we know, no literature is available about the adrenal ultrastructure in the envenoming syndrome caused by scorpion or bee venom, neither in human nor in animal models. In this work we report the ultrastructural alterations in adrenal glands of mice inoculated with scorpion (Tytius discrepans) and bee (Apis melifera) venoms.

Adult male mice were intraperitoneally injected with a lethal dose fifty $\left(\mathrm{LD}_{50}\right)$ of $T$. discrepans venom $(0.5 \mathrm{mg} / \mathrm{kg})$ or with a $\mathrm{LD}_{50}(5 \mu \mathrm{g} / 20 \mathrm{~g})$ of $A$. melifera venom. Control mice received saline solution $(0.1 \mathrm{ml})$ by the same route. Adrenal gland samples were obtained from animals sacrificed at different time periods after venom inoculation, and processed with routine techniques for transmission electron microscopy. Sections were observed in Hitachi H-300 and H-500 electron microscopes.

All mice injected with venom showed the typical clinical manifestations of envenoming. In mice inoculated with $T$. discrepans venom, the most relevant adrenal changes were seen at 48 hours (Fig 1). These changes included mitochondria and smooth endoplasmic reticulum generalized swelling in the majority of cortex cells observed. Autophagic vacuoles containing mitochondrial residues were also seen (Fig. 1). In mice injected with $A$. melifera venom, a remarkable disruption of smooth endoplasmic reticulum and capillaries with widened fenestrae, were observed at 6 hours after venom inoculation (Fig. 2). At $48 \mathrm{~h}$ after inoculation with this venom, the abnormalities observed included swelling of mitochondria, autophagic vacuoles with mitochondrial rests (Fig. 3) and changes in microvasculature.

Our results demonstrate that adrenal gland cortex profound damage may result from the action of toxins present in both, scorpion and bee venom. The lesions we found in mitochondria and endoplasmic reticulum might be sufficient to cause impairment of steroid synthesis. These findings suggest that different degrees of suprarenal dysfunction could be present in humans exhibiting the clinical manifestation of envenoming above described.

○ [1] M. Ismail, Toxicon 33 (1995) 825.

$\circ$ [2] A. Machado and A. Rodríguez-Acosta, Los Animales venenosos y ponzoñosos de Venezuela, CDCH-UCV, Caracas, 1998. 

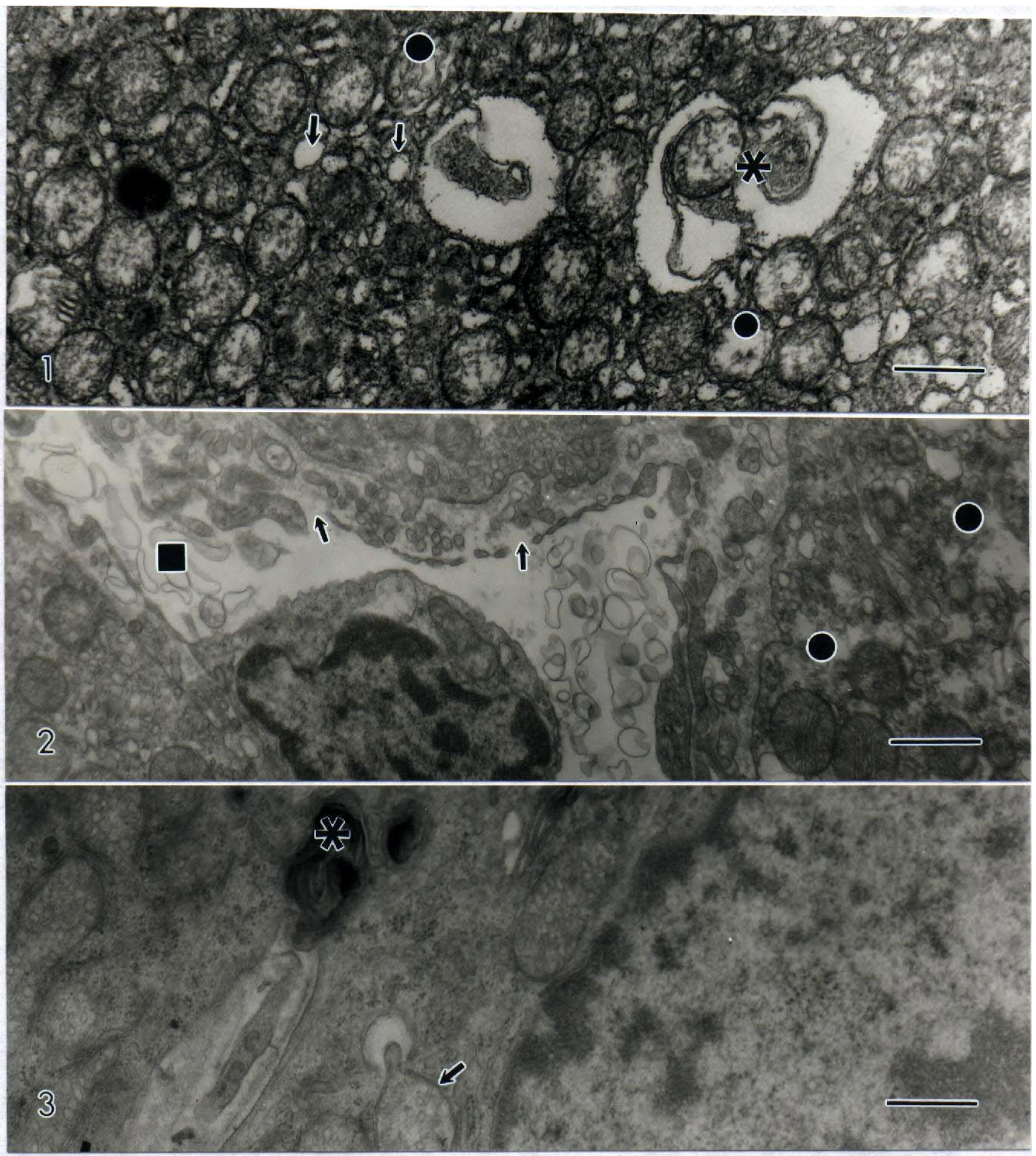

Fig. 1. T. discrepans inoculated mice. Autophagic vacuole (asterisk), swelling of smooth endoplasmic reticulum (arrows) and mitochondria (circles). Bar $=0.7 \mu \mathrm{m}$.

Fig. 2. A. melifera inoculated mice. Smooth endoplasmic reticulum disruption (circles). Capillary with widened fenestrae (arrows), and membranous debris into the lumen (square). Bar $=0.7 \mu \mathrm{m}$. Fig. 3. A. melifera inoculated mice.Autophagic vacuole (asterisk).Swollen mitochondrion (arrow). $\mathrm{Bar}=0.5 \mu \mathrm{m}$. 